\title{
Frecuencia de los tipos de infarto agudo de miocardio según la tercera definición
}

\author{
Paola Calvachi Prieto ${ }^{a, b}$, Dormar David Barrios ${ }^{c}$, Mariana Puccini ${ }^{\mathrm{b}}$, \\ Henry Alberto Mojica ${ }^{b}$, David Delgadillo ${ }^{a, b}$, Mabel Gómez ${ }^{a, c}$, Edgar Celis ${ }^{a}$ \\ y Andrés Felipe Buitrago ${ }^{a, c, *}$
}

\author{
a Departamento de Medicina Crítica y Cuidado Intensivo, Hospital Universitario Fundación Santa Fe de Bogotá, Bogotá, Colombia \\ b Universidad de los Andes, Facultad de Medicina, Bogotá, Colombia \\ ' Departamento de Medicina Interna, sección de Cardiología, Hospital Universitario Fundación Santa Fe de Bogotá, Bogotá, \\ Colombia
}

Recibido el 1 de mayo de 2016; aceptado el 13 de junio de 2017

Disponible en Internet el 20 de noviembre de 2017

\author{
PALABRAS CLAVE \\ Enfermedad \\ cardiovascular; \\ Isquemia; \\ Infarto de miocardio
}

\begin{abstract}
Resumen
Introducción y objetivos: El infarto agudo de miocardio (IAM) es una patología muy prevalente con una morbimortalidad significativa en Colombia y en el mundo. Es por esto que desde el 2011 en la Fundación Santa Fe de Bogotá se inició la recolección de datos demográficos y clínicos de pacientes con patologías cardiovasculares en una base de datos denominada Registro Colombiano de Enfermedades Cardiovasculares. Actualmente se tienen 736 registrados según el tipo de IAM que presentan de acuerdo con la tercera clasificación de infarto. Por lo anterior, se quiso conocer la frecuencia de cada tipo de infarto en nuestra población y su asociación con diferentes variables.

Métodos: Se realizó un estudio descriptivo observacional de corte transversal en el cual se revisaron los datos de los pacientes pertenecientes a RECODEC y se evaluó el tipo de IAM que presentaban.

Resultados: Se encontró alta frecuencia para el infarto tipo 1, intermedia para el tipo 2 y baja para los otros tipos de infarto, además una asociación positiva entre ser hombre y presentar infarto tipo 1 ( $\mathrm{n}=427,85,7 \%$ con un OR 1,91 IC95\% 1,29-2,82) y una asociación entre infarto tipo 2 y ser mujer ( $n=51,21,4 \%$ con un OR 0,51 IC95\% $0,33-0,76)$.

Conclusiones: Una adecuada clasificación del tipo de infarto permite definir una mejor ruta terapéutica para el paciente y predecir posibles complicaciones. Con este estudio se logró realizar la caracterización de la población en cada grupo establecido por la tercera definición. (C) 2017 Sociedad Colombiana de Cardiología y Cirugía Cardiovascular. Publicado por Elsevier España, S.L.U. Este es un artículo Open Access bajo la licencia CC BY-NC-ND (http:// creativecommons.org/licenses/by-nc-nd/4.0/).
\end{abstract}

\footnotetext{
* Autor para correspondencia.

Correo electrónico: abuitrag@uniandes.edu.co (A.F. Buitrago).
} 


\section{KEYWORDS}

Cardiovascular

disease;

Ischaemia;

Myocardial infarction

\section{Frequency of myocardial infarction according to the third universal definition}

\begin{abstract}
Introduction and objectives: Acute myocardial infarction is a disease associated with a high prevalence and morbidity in Colombia and worldwide. Since 2011, at the Fundación Santa Fe de Bogotá, a registry known as RECODEC began to be used to collect data in order to characterise the population with cardiovascular diseases. A total of 736 patients have currently been registered according to the type of myocardial infarction, taking into account its third universal definition. The application of this definition has been related with an increase in the number of cases diagnosed, with prediction of 10 year mortality, and with improvement of care. The prevalence is presented of each type of infarction and its association with different kind of variables.

Methods: A descriptive, observational, cross sectional study was conducted using the patient data from RECODEC registry, in order to evaluate the type of infarction each patient presented. Results: A high prevalence was found for type 1 infarction, with intermediate for type 2 , and low for the other types of myocardial infarction. A positive association was also found between being male and having a type 1 infarction ( $\mathrm{n}=427,85.7 \%$ and OR: $1.91,95 \% \mathrm{cl}: 1.29-2.82, \mathrm{P}<.001)$ and an association between being female and type 2 infarction $(n=51,21.4 \%$ and OR: $0.51,95 \%$ $\mathrm{Cl}$ : 0.33-0.76, $\mathrm{P}<.001)$.

Conclusions: It is important to classify the type of infarction presented by the patients, so that the type of population that suffers from each type could be characterised, so that it may be possible to offer a more specific treatment for each disease.

(c) 2017 Sociedad Colombiana de Cardiología y Cirugía Cardiovascular. Published by Elsevier España, S.L.U. This is an open access article under the CC BY-NC-ND license (http:// creativecommons.org/licenses/by-nc-nd/4.0/).
\end{abstract}

\section{Introducción}

El infarto agudo de miocardio es una de las patologías con mayor prevalencia en la actualidad debido a su gran morbimortalidad. Según datos del año 2015 aportados por la Sociedad Americana del Corazón (AHA), los centros de control y prevención de enfermedades y los Institutos Nacionales de Salud la prevalencia de infarto agudo de miocardio en hombres de Estados Unidos se encuentra entre 11,3\% $17,3 \%$ en pacientes mayores de 60 años y entre $4,2 \%$ y $8,9 \%$ en mujeres del mismo grupo etario ${ }^{1-5}$.

Desde su primera publicación en 1971, la Organización Mundial de la Salud ha buscado la mejor forma de definir esta entidad con fines diagnósticos, epidemiológicos e investigativos ${ }^{4}$. Por esto, a lo largo de este tiempo han surgido diferentes clasificaciones, entre las que se encuentra la tercera definición de infarto ${ }^{1}$, documento en el cual Thygesen et al. ${ }^{6}$ clasificaron el infarto agudo de miocardio en cinco tipos:

a. Tipo 1: infarto agudo de miocardio espontáneo.

b. Tipo 2: infarto agudo de miocardio secundario a desequilibrio isquémico.

c. Tipo 3: infarto agudo de miocardio que conduce a muerte cuando aún no se dispone de los resultados de biomarcadores.

d. Tipo 4A: infarto agudo de miocardio relacionado con intervención coronaria percutánea.

e. Tipo 4B: infarto agudo de miocardio relacionado con trombosis del stent.

f. Tipo 5: infarto agudo de miocardio relacionado con derivación aorto-coronaria con injerto.
Con base en lo anterior se estudió la frecuencia de cada tipo de infarto en un hospital de cuarto nivel de Bogotá, a fin de generar asociaciones con diferentes variables.

\section{Métodos}

Se llevó a cabo un estudio descriptivo, observacional, retrospectivo, de corte transversal en el que se incluyeron 736 pacientes con diagnóstico de infarto agudo de miocardio que ingresaron al Hospital Universitario Fundación Santa Fe de Bogotá entre enero de 2011 y enero de 2016. El estudio inició una vez se contó con la validación de la base de datos perteneciente al Registro Colombiano de Enfermedades Cardiovasculares (RECODEC), por parte de los investigadores ${ }^{7}$.

Se hizo una revisión detallada de los datos recolectados y se obtuvo un registro con el conteo del total de pacientes con diagnóstico de infarto agudo de miocardio en 5 años (el diagnóstico se elaboró por historia clínica, en la cual se valoraba al paciente por parte de Cardiología, valores de troponina y cambios electrocardiográficos). Una vez obtenido el número total de pacientes se calculó la frecuencia de cada tipo de infarto y se realizó un análisis bivariado entre tipo de infarto y las siguientes variables: sexo, edad, antecedente de dislipidemia, obesidad, hipertensión arterial, infarto previo y tabaquismo.

El diagnóstico de pacientes con infarto tipo 2 fue tomado directamente de la historia clínica.

\section{Análisis estadístico}

Los resultados se analizaron con el software SPSS statistics versión 23 y Epidat 3.1. Las variables cuantitativas se pre- 
Tabla 1 Caracterización de la población por sexo

\begin{tabular}{lll}
\hline Sexo & Frecuencia & Porcentaje \\
\hline Hombre & 498 & 67,7 \\
Mujer & 238 & 32,3 \\
Total & 736 & 100 \\
\hline
\end{tabular}

sentan con medidas de tendencia central y de dispersión, en tanto que las variables cualitativas con frecuencias y porcentajes. Adicionalmente, se efectuó un análisis bivariado con el fin de encontrar asociaciones y distribución de probabilidad. Se consideró el valor de $\mathrm{p}$ menor de 0,05 como estadísticamente significativo.

El análisis bivariado se realizó teniendo en cuenta las categorías de edad estipuladas y el género de los pacientes, respectivamente.

\section{Resultados}

Se obtuvieron datos de 736 pacientes, de los cuales 498 eran hombres $(67,7 \%)$ y 238 mujeres $(32,3 \%)$, que se encontraban registrados en RECODEC. El promedio de edad fue de 65,8 años con un rango entre 19 y 98 años (tablas 1 y 2).

Se analizó la asociación de la variable edad con la variable tipo de infarto sin encontrar resultados estadísticamente significativos $(p=0,56)$. A continuación se agruparon en cuatro categorías (19 a 39 años; 40 a 59 años; 60 a 79 años; 80 a 99 años) y se analizó si existía asociación con la variable tipo de infarto, sin hallar asociación estadísticamente significativa por la prueba de chi cuadrado $(p=0,44)$.

Según los resultados reportados y la clasificación, el tipo de infarto más frecuente fue el 1 (607 pacientes, 82,5\%), seguido por el 2 (111 pacientes, 15,1\%), el 4B (7 pacientes, $1 \%)$, el 3 ( 6 pacientes, $0,8 \%$ ), el 5 (4 pacientes, $0,5 \%)$ y finalmente el 4A (5 pacientes, 0,1\%) (tabla 3).

Para la relación entre el tipo de infarto y el sexo de los pacientes se encontró una frecuencia mayor de mujeres en el infarto agudo de miocardio tipo 3 (4 mujeres vs. 2 hombres) y $4 \mathrm{~A}$ ( 1 mujer vs. 0 hombres). En los demás tipos de infarto se observó mayor frecuencia en hombres (tipo 1: 427 hombres vs. 180 mujeres; tipo 2: 60 hombres vs. 51 mujeres; tipo 4B: 6 hombres vs. 1 mujer y tipo 5: 3 hombres vs. 1 mujer) (tabla 4).
Al cruzar todos los tipos de infarto con el sexo de los pacientes que cumplieron con los criterios de inclusión, se evidencia que existe asociación estadísticamente significativa entre el tipo 1 y 2 de infarto y el sexo, asociación positiva entre ser hombre e infarto tipo $1(\mathrm{n}=427,85,7 \%$, OR 1,91 IC95\% 1,29-2,82) y asociación entre infarto tipo 2 y ser mujer $(n=51,21,4 \%$, OR 0,51 IC $95 \% 0,33-0,76)$. Con los demás tipos de infarto y sexo no se encontraron asociaciones significativas. Sin embargo, al evaluar las frecuencias de aparición de los demás tipos de infarto se aprecia que la muestra es pequeña en esos casos (tabla 3 ).

Adicional a la edad y al sexo, se evaluaron las asociaciones entre tipo de infarto y antecedente de dislipidemia, hipertensión arterial, obesidad, tabaquismo e infarto previo. Sin embargo, para ninguna de estas variables se encontró una relación estadísticamente significativa.

\section{Discusión}

Este estudio de investigación permite brindar un acercamiento al panorama colombiano respecto a la frecuencia de los subtipos de infarto agudo de miocardio en la población bogotana, con el fin de ser comparados con datos epidemiológicos de otros países.

En el análisis bivariado de género (femenino y masculino) con las demás variables se encontró que el $85,7 \%$ de los hombres presentan infarto tipo 1, comparado con el grupo de mujeres, en el que el 21,4\% presentan infarto tipo 2. En el VIRGO $^{8}$ (Variation in Recovery: Role of Gender on Outcomes of Young AMI Patients), un estudio prospectivo observacional multicéntrico con jóvenes de ambos géneros entre 18-55 años (media $47 \pm 6$ años), se demostró que el sexo femenino tiene alta frecuencia de factores de riesgo cardiovasculares, comorbilidades y factores psicosociales en comparación con el género masculino. Además, se demostró que las mujeres debutan con una presentación clínica atípica, asociada a un alto riesgo de desenlaces fatales posterior a un infarto agudo de miocardio. Por otro lado, se demostró que las mujeres de mediana edad con infarto agudo de miocardio representan una población con mayor carga de enfermedad comparadas con hombres de su misma edad. Esto significa que el hecho de ser mujer por sí solo, confiere alto riesgo de enfermedad cardiovascular.

Tabla 2 Distribución de tipo de infarto por grupo de edad

\begin{tabular}{|c|c|c|c|c|c|c|c|c|c|}
\hline \multirow{3}{*}{$\begin{array}{l}\text { Tipo de } \\
\text { infarto }\end{array}$} & \multicolumn{8}{|c|}{ Edad } & \multirow{3}{*}{$\begin{array}{l}\text { Total } \\
\text { (n) }\end{array}$} \\
\hline & \multicolumn{2}{|c|}{ Entre 19 y 39 años } & \multicolumn{2}{|c|}{ Entre 40 y 59 años } & \multicolumn{2}{|c|}{ Entre 60 y 79 años } & \multicolumn{2}{|c|}{ Entre 80 y 99 años } & \\
\hline & $\mathrm{N}$ & $\%$ & $\mathrm{n}$ & $\%$ & $\mathrm{n}$ & $\%$ & $\mathrm{n}$ & $\%$ & \\
\hline 1 & 13 & 2 & 198 & 33 & 280 & 46 & 116 & 19 & 607 \\
\hline 2 & 7 & 6 & 30 & 27 & 51 & 46 & 23 & 21 & 111 \\
\hline 3 & 0 & 0 & 2 & 33 & 3 & 50 & 1 & 17 & 6 \\
\hline 4 & 0 & 0 & 4 & 50 & 4 & 50 & 0 & 0 & 8 \\
\hline 5 & 0 & 0 & 0 & 0 & 3 & 75 & 1 & 25 & 4 \\
\hline Total & 20 & 2,7 & 234 & 31,8 & 341 & 46,3 & 141 & 19,2 & 736 \\
\hline
\end{tabular}

*Por cada tipo de infarto se presenta el porcentaje que corresponde a cada grupo etario. En la última fila, los porcentajes corresponden a la proporción del total de la población. 
Tabla 3 Distribución por tipo de infarto

\begin{tabular}{|c|c|c|}
\hline $\begin{array}{l}\text { Tipos de } \\
\text { infarto }\end{array}$ & $\begin{array}{l}\text { Frecuencia } \\
\mathrm{n}\end{array}$ & $\begin{array}{l}\text { Porcentaje } \\
\%\end{array}$ \\
\hline 1 & 607 & 82,5 \\
\hline 2 & 111 & 15,1 \\
\hline 3 & 6 & 0,8 \\
\hline $4 \mathrm{~A}$ & 1 & 0,1 \\
\hline $4 B$ & 7 & 1 \\
\hline 5 & 4 & 0,5 \\
\hline Total & 736 & 100 \\
\hline
\end{tabular}

Este análisis había sido propuesto por grupos como el de Beck, et $\mathrm{al}^{9}$; Garavalia, et $\mathrm{al}^{10}$ y Schweikert, et al. ${ }^{11}$, los cuales demostraron que mujeres menores de 55 años tienen dos a tres veces más riesgo de mortalidad intrahospitalaria después de un infarto agudo de miocardio y un $50 \%$ de riesgo de muerte a los dos años, en comparación con el género masculino de igual edad.

Desde el punto de vista fisiopatológico, el estudio WISE $^{12,13}$ (Women's Ischemia Syndrome Evaluation) afianzó la teoría de que el sexo femenino presenta más enfermedad coronaria difusa y menos lesiones obstructivas que los hombres. Por otra parte, tienen arterias coronarias menos estrechas y son más propensas a enfermedad coronaria microvascular o disección coronaria espontánea.

Respecto al infarto agudo de miocardio tipo 2, autores como Alpert y Thyegesen ${ }^{14}$, comentan que en las más recientes definiciones, la novedad ha sido la selección de un grupo de pacientes con infarto agudo de miocardio sin enfermedad coronaria obstructiva, quienes conllevarían un manejo diferente. Sin embargo, respecto a lo anterior, los autores promueven la posibilidad que en próximas definiciones al infarto tipo 2 se le debería llamar lesión miocárdica, ya que muchos de estos individuos tienen comorbilidades que pueden desencadenar enfermedad cardiovascular, pero sin cambios de gran importancia en cuanto a la suplencia y demanda de oxígeno.

Nagele ${ }^{15}$, quien afirma en su publicación que se deben integrar ambos términos (infarto agudo de miocardio tipo 2 + lesión miocárdica) en uno solo:lesión miocárdica aguda. Sin embargo, la no presencia de este diagnóstico o término patológico en la Clasificación Internacional de Enfermedades en su décima revisión impide su uso de manera rutinaria en el ámbito clínico e investigativo.

En lo que concierne a la frecuencia de presentación de pacientes con infarto agudo de miocardio tipo 2 , se reportó una frecuencia del $15,1 \%$, lo cual demuestra que esta patología es multifactorial y noxas como anemia, hipoxia, enfermedad coronaria estable y metabólicas, entre otras, pueden ocasionar sintomatología y clínica similar a la del infarto agudo de miocardio tipo $1^{16}$.

El estudio de Bonaca, et al. en el que se reclutaron 96 pacientes mayores de 18 años con dolor torácico típico y medición de biomarcadores al ingreso, a las 4-6 horas y a las 12-24 horas posteriores al dolor, mostró una frecuencia para infarto agudo de miocardio tipo 1 de $90 \%$, y sólo del $10 \%$ para el tipo 2, cifras que concuerdan con los resultados obtenidos en nuestro estudio $(82,5 \%$ y de infarto agudo de miocardio tipo $215,1 \%)^{17}$.

La ausencia de pruebas definitivas no invasivas para diferenciar entre infarto agudo de miocardio tipos 1 y 2 , torna especulativo el diagnóstico del segundo. Por esto, autores como Thygesen et al. ${ }^{18,19}$, Alpert et al. ${ }^{20}$, Saaby et al. ${ }^{21}$, Javed et al. ${ }^{22}$ y González et al. ${ }^{23}$, afirman que la frecuencia oscila alrededor del $1,6 \%$ y $29,6 \%$, lo cual es de esperar ya que en la práctica clínica puede ser difícil distinguir entre infarto tipos 1 y 2 , al igual que otras condiciones no isquémicas que pueden provocar daño miocárdico y elevación de biomarcadores.

Otro aporte interesante lo publicó el grupo de trabajo de Baron et al. ${ }^{24}$, en el que la media de edad de la población con infarto agudo de miocardio tipo 2 era mayor, al compararse con los pacientes con infarto agudo de miocardio tipo 1. Al cotejar estos resultados con los de nuestro estudio, el porcentaje de personas mayores de 60 años en los dos grupos (infarto agudo de miocardio tipos 1 y 2) se encontraba entre el $67-70 \%$. Adicionalmente, fue raro pero no totalmente ausente, encontrar elevación del segmento ST en los pacientes con infarto agudo de miocardio tipo 2 , en tanto que fue más común la presencia de bloqueo de rama izquierda de reciente aparición en esta población ${ }^{24}$.

En cuanto al infarto agudo de miocardio tipo 3, se reportó una frecuencia relativamente baja, del 0,8\%, al compararla con los datos publicados por Javed et al. quienes reportaron un $4,2 \%^{22}$.

Respecto a la población diagnosticada con infarto agudo de miocardio tipos 4 A, 4 B y 5 , el porcentaje fue $0,1 \%, 1 \%$ y $0,5 \%$ respectivamente, teniendo como criterio diagnóstico el valor de troponina, criterios electrocardiográficos, imágenes e identificación intracoronaria de trombosis ${ }^{1}$. Es importante mencionar que esta frecuencia al igual que la de los tipos anteriores, concuerda con datos obtenidos según la revisión de la literatura realizada por Sandoval et al. ${ }^{14}$. Sin embargo, se requiere, según este autor y su grupo de investi-

Tabla 4 Asociación entre sexo y tipo de infarto

\begin{tabular}{|c|c|c|c|c|c|c|c|}
\hline \multirow{2}{*}{$\begin{array}{l}\text { Sexo } \\
\text { Tipo de infarto }\end{array}$} & \multicolumn{2}{|c|}{ Hombre } & \multicolumn{2}{|c|}{ Mujer } & \multicolumn{3}{|c|}{ Estadístico } \\
\hline & $\mathrm{n}$ & $\%$ & $\mathrm{n}$ & $\%$ & Chi-cuadrado & OR & IC 95\% \\
\hline 1 & 427 & 85,7 & 180 & 75,6 & $<0,001$ & 1,91 & $1,29-2,82$ \\
\hline 2 & 60 & 12,0 & 51 & 21,4 & $<0,001$ & 0,51 & $0,33-0,76$ \\
\hline 3 & 2 & 0,4 & 4 & 1,7 & 0,07 & 0,24 & $0,04-1,31$ \\
\hline $4 \mathrm{~B}$ & 6 & 1,2 & 1 & 0,4 & 0,28 & 2,89 & $0,34-24,14$ \\
\hline 5 & 3 & 0,6 & 1 & 0,4 & 0,61 & 1,43 & $0,14-13,8$ \\
\hline
\end{tabular}

*Tipo 4A no se describe porque el valor fue 0 en la categoría hombres. 
gación, mayor evidencia para la reproducción de guías para pacientes con esta patología.

\section{Limitaciones del estudio}

Los resultados reportados permiten esclarecer las frecuencia de cada tipo de infarto de los pacientes que ingresaron a urgencias de la Fundación Santa Fe de Bogotá desde el 2011 hasta el 2016. Aunque la muestra es significativa, se debe seguir con la recolección de los datos para poder contar con una muestra más grande en la frecuencia de los tipos de infarto 3, 4A, 4B y 5, dado que los casos reportados siguen siendo pocos para poder hacer asociaciones con otras variables.

Por otra parte, para los pacientes con diagnóstico de infarto agudo de miocardio tipo 2 no fue posible definir la enfermedad a la cual se le atribuye la lesión cardiaca, dado que esa información no pudo ser obtenida de todas las historias clínicas revisadas.

Es preciso tener en cuenta que por el diseño del estudio puede haber un sub-registro de datos, ya que si Cardiología no solicita el seguimiento, estos no ingresan al registro de la base de datos. En el caso de pacientes con infarto tipo 3 , los datos se pierden en el servicio de urgencias, mientras que para pacientes con infartos tipo 2 diagnosticados en unidades de cuidado intensivo, puede no ser solicitado el seguimiento por Cardiología. Por último, en pacientes con infarto tipo 4 y 5 , ante ausencia de síntomas, no hay seguimiento enzimático ni electrocardiográfico.

\section{Conclusiones}

La patología cardiovascular y sobre todo el Infarto agudo de miocardio, se ha convertido en una de las patologías más estudiadas en las últimas décadas, tanto por su incidencia, como por la prevalencia de los diferentes factores de riesgo implicados en su aparición. Es por esto que en la actualidad se han creado múltiples iniciativas mundiales que buscan como estrategia final el pronto diagnóstico y manejo médico para prevenir todas sus complicaciones y disminuir una mortalidad que viene en aumento.

El desarrollo de estudios como este, permite la caracterización y validación adecuadas de la definición de infarto agudo de miocardio estandarizada de manera mundial, en una población como la nuestra, que se vuelve cada vez más proclive a todo tipo de patologías cardiovasculares, ante todo al infarto agudo de miocardio. Todos estos esfuerzos buscan promover la prevención y promoción en salud desde los primeros niveles de atención, así como ayudar a mitigar los costos en salud.

Mediante el conocimiento de la estadística local y regional se podrán afianzar programas estatales y privados para la valoración y tratamiento correctos de esta patología en particular.

La clasificación apropiada de la patología en infarto agudo de miocardio, ciñéndose a la tercera definición universal de infarto, es crucial ya que de esta manera es posible direccionar los esfuerzos hacia la población que con mayor frecuencia presenta cada tipo de infarto y así brindar con mayor facilidad un tratamiento específico para cada uno.
De otro lado, sugiere adelantar estudios en enfermedades trazadoras con poblacionales mayores que permitan identificar factores de riesgo, con el objetivo de mitigarlos a tiempo. Así mismo, conviene establecer más relaciones con otras variables que permitan analizar de manera profunda el porqué de los resultados obtenidos y encontrar factores de riesgo que permitan anticiparse a la aparición del infarto.

Este estudio, en concordancia con la literatura revisada, demostró que el tipo de infarto más frecuente fue el 1, alrededor del $82,5 \%$, seguido por el $2(15,1 \%)$; sin embargo, como se expuso anteriormente, es claro que urge redefinir y diferenciar la definición de infarto agudo de miocardio como tal de una lesión miocárdica aguda, en la que se tengan en cuenta nuevos estudios y evidencia que deba ser publicada y revisada. El resto de la población correspondió a los otros tipos de infarto, lo cual guarda relación con el panorama científico mundial, de cara a datos similares y próximos a los arrojados por nuestro estudio.

El sexo femenino sigue siendo, al igual que en otras patologías, un factor de riesgo predominante en la enfermedad cardiovascular, sobre todo en el infarto tipo 2, evidenciándose una asociación entre el infarto tipo 1-2 y el sexo. Es más común la presentación del tipo 1 en los hombres y la de tipo 2 en las mujeres.

Como se expresó antes, será necesario recolectar más datos de futuras publicaciones, al igual que nuevas definiciones con nuevos términos que estén al alcance del médico, para poder estudiar a profundidad una patología que día a día aumenta en incidencia y prevalencia. Queda continuar con estudios e investigaciones en el área de salud pública y bioestadística para poder enfrentar las diferentes presentaciones de esta patología, al parecer de nunca acabar.

\section{Responsabilidades éticas}

Protección de personas y animales. Los autores declaran que para esta investigación no se han realizado experimentos en seres humanos ni en animales.

Confidencialidad de los datos. Los autores declaran que han seguido los protocolos de su centro de trabajo sobre la publicación de datos de pacientes.

Derecho a la privacidad y consentimiento informado. Los autores declaran que en este artículo no aparecen datos de pacientes.

\section{Conflictos de interés}

Ninguno.

\section{Bibliografía}

1. White HD, Thygesen K, Alpert JS, Jaffe AS. Clinical implications of the Third Universal Definition of Myocardial Infarction. Heart. 2014;100:424-32.

2. Sandoval Y, Smith SW, Schulz KM, Murakami MM, Love SA, Nicholson J, et al. Diagnosis of type 1 and type 2 myocardial infarction using a high-sensitivity cardiac troponin $i$ assay with sex-specific $99^{\text {th }}$. percentiles based on the third Universal Definition of 
Myocardial Infarction Classification System. Clinical Chemistry. 2015;61:657-63.

3. Jaffe AS, Apple FS. The Third Universal Definition of Myocardial Infarction-Moving Forward. Clinical Chemistry. 2012;58:1727-8.

4. Jneid H, Alam M, Virani SS, Bozkurt B. Redefining myocardial infarction: what is new in the ESC/ACCF/AHA/WHF Third Universal Definition of myocardial infarction? MDCVJ. 2013;9:169-72.

5. Mozaffarian D, Benjamin EJ, Go AS, Amett DK, Blaha MJ, Cushman M, et al. Heart Disease and Stroke Statistics-2015 Update A Report From the American Heart Association. Circulation. 2015;131:e29-322.

6. Thygesen K, Alpert JS, Jaffe AS, Simoons ML, Chaitman BR, White HD. Documento de consenso de expertos. Tercera definición universal del infarto de miocardio. Rev Esp Cardiol. 2013;66:e1-15.

7. Soto M, Buitrago AF, Gómez M, Celis E. Indicadores de calidad en la atención de pacientes con infarto agudo de miocardio. Rev Colomb Cardiol. 2014;21:301-7.

8. Bucholz EM, Strait MK, Dreyer PR, Lindau TS, DÓnofrio G, Geda $M$, et al. Sex differences in young patients with acute myocardial infarction: A VIRGO study analysis. Eur Heart J Acute Cardiovasc Care. 2017;6:610-22.

9. Beck CA, Joseph L, Bélisle P, Pilote L. QOLAMI Investigators (Quality of life in acute myocardial infarction). Predictors of quality of life 6 months and 1 year after acute myocardial infarction. Am Heart J. 2001;142:271-9.

10. Garavalia LS, Decker C, Reid KJ, Lichtman JH, Parashar S, Vaccarino $\mathrm{V}$, et al. Does health status differ between men and women in early recovery after myo- cardial infarction? J Womens Health. 2007;16:93-101.

11. Schweikert B, Hunger M, Meisinger C, Konig HH, Gapp O, Holle R. Quality of life several years after myocardial infarction: Comparing the MONICA/KORA registry to the general population. Eur Heart J. 2009;30:436-43.

12. Quyyumi AA. Women and ischemic heart disease: Pathophysiologic implications from the Women's Ischemia Syndrome Evaluation (WISE) study and future research steps. J Am Coll Cardiol. 2006;47:566-71.

13. Bairey Merz CN, Shaw LF, Reis SE, Bittner V, Olson M, Johnson $\mathrm{BD}$, et al. Insights from the NHLBI-sponsored Women's Ischemia Syndrome Evaluation (WISE) study: Part II: Gender differences in presentation, diagnosis, and outcome with regard to gender-based pathophysiology of atherosclerosis and macrovas- cular and microvascular coronary disease. J Am Coll Cardiol. 2006;47:521-9.

14. Alpert JS, Thygesen KA. The case for a revised definition of myocardial infarction-the ongoing conundrum of type 2 myocardial infarction vs. myocardial injury. JAMA Cardiol. 2016;1: 249-50.

15. Nagele P. The case for a revised definition of myocardial infarction-resolving the ambiguity of type 2 myocardial infarction. JAMA Cardiol. 2016;1:247-8.

16. Sandoval Y, Smith SW, Thordsen SE, Apple FS. Supply/Demand Type 2 Myocardial Infarction: Should We Be Paying More Attention? J Am Coll Cardiol. 2014;63:2079-87.

17. Bonaca MP, Ruff CT, Kosowsky J, Conrad MJ, Murphy SA, Sabatine MS, et al. Evaluation of the diagnostic performance of current and next-generation assays for cardiac troponin I in the BWHTIMI ED Chest Pain Study. Eur Heart J Acute Cardiovasc Care. 2013;2:195-202.

18. Thygesen K, Alpert JS, White HD. Joint ESC/ACCF/AHA/WHF Task Force for the Redefinition of Myocardial Infarction. Universal definition of myocardial infarction. Eur Heart J. 2007;28:2525-38.

19. Thygesen K, Alpert JS, Jaffe AS, Simoons ML, Chaitman BR, White HD, et al. JointESC/ACCF/AHA/WHF Task Force for Universal Definition of Myocardial Infarction. Third universal definition of myocardial infarction. J Am Coll Cardiol. 2012;60:1581-98.

20. Alpert JS, Thygesen KA, White HD, Jaffe AS. Diagnostic and therapeutic implications of type 2 myocardial infarction: review and commentary. Am J Med. 2014;127:105-8.

21. Saaby L, Poulsen TS, Hosbond S, Larsen T.B., Pyndt Diederichsen AC, Hallas J, et al. Classification of myocardial infarction: frequency and features of type 2 myocardial infarction. Am J Med. 2013;126:789-97.

22. Javed U, Aftab W, Ambrose JA, Wessel RJ, Mouanoutoua M, Huang G, et al. Frequency of elevated troponin I and diagnosis of acute myocardial infarction. Am J Cardiol. 2009;104:9-13.

23. Gonzalez MA, Eilen DJ, Marzouq RA, Porterfield CP, Hazarika S, Nasir S, et al. Multidisciplinary Atherosclerosis Prevention Program. The universal classification is an independent predictor of long-term outcomes in acute myocardial infarction. Cardiovasc Revasc Med. 2011;12:35-40.

24. Baron T, Hambraeus K, Sundström J, Erlinge D, Jernberg T, Lindahl B, TOTAL-AMI study group. Type 2 myocardial infarction in clinical practice. Heart. 2015;101:101-6. 